\title{
Improved Leakage Correction for Single-Echo Dynamic Susceptibility Contrast Perfusion MRI Estimates of Relative Cerebral Blood Volume in High-Grade Gliomas by Accounting for Bidirectional Contrast Agent Exchange
}

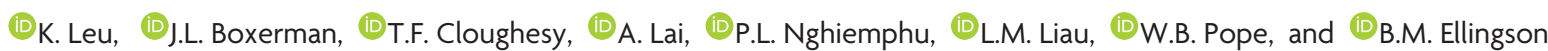

\begin{abstract}
BACKGROUND AND PURPOSE: Contrast agent extravasation through a disrupted blood-brain barrier potentiates inaccurate DSC MR imaging estimation of relative CBV. We explored whether incorporation of an interstitial washout rate in a leakage-correction model for single-echo, gradient-echo DSC MR imaging improves relative CBV estimates in high-grade gliomas.
\end{abstract}

\begin{abstract}
MATERIALS AND METHODS: We modified the traditional model-based postprocessing leakage-correction algorithm, assuming unidirectional contrast agent extravasation (Boxerman-Weisskoff model) to account for bidirectional contrast agent exchange between intraand extravascular spaces (bidirectional model). For both models, we compared the goodness of fit with the parent leakage-contaminated relaxation rate curves by using the Akaike Information Criterion and the difference between modeled interstitial relaxation rate curves and dynamic contrast-enhanced MR imaging by using Euclidean distance in 21 patients with glioblastoma multiforme.

RESULTS: The bidirectional model had improved Akaike Information Criterion versus the bidirectional model in $>50 \%$ of enhancing tumor voxels in all 21 glioblastoma multiformes $(77 \% \pm 9 \% ; P<.0001)$ and had reduced the Euclidean distance in $>50 \%$ of enhancing tumor voxels for $17 / 21$ glioblastoma multiformes $(62 \% \pm 17 \% ; P=.0041)$. The bidirectional model and dynamic contrast-enhanced-derived $k_{\text {ep }}$ demonstrated a strong correlation $(r=0.74 \pm 0.13)$. On average, enhancing tumor relative CBV for the Boxerman-Weisskoff model exceeded that for the bidirectional model by $16.6 \% \pm 14.0 \%$.
\end{abstract}

CONCLUSIONS: Inclusion of the bidirectional exchange in leakage-correction models for single-echo DSC MR imaging improves the model fit to leakage-contaminated DSC MR imaging data and significantly improves the estimation of relative CBV in high-grade gliomas.

ABBREVIATIONS: $\mathrm{AIC}=$ Akaike Information Criterion; bidir model $=$ bidirectional model; $\mathrm{BW}$ model $=$ Boxerman-Weisskoff model; $\Delta R_{2}{ }^{*}=$ transverse relaxation rate; $\mathrm{DCE}=$ dynamic contrast-enhanced; $\mathrm{GBM}=$ glioblastoma multiforme; $\mathrm{k}_{\mathrm{ep}}=$ transfer constant from the extracellular extravascular space back to the blood plasma; $K^{\text {trans }}=$ contrast transfer coefficient; $r C B V=$ relative cerebral blood volume

$\mathbf{T}$ he most common DSC MR imaging metric in neuro-oncology is relative $\mathrm{CBV}(\mathrm{rCBV}),{ }^{1}$ which has been used for grading gliomas, ${ }^{2,3}$ predicting low-grade to high-grade transformation, ${ }^{4,5}$

Received September 5, 2015; accepted after revision January 28, 2016

From the UCLA Brain Tumor Imaging Laboratory (K.L., B.M.E.), Center for Computer Vision and Imaging Biomarkers; Department of Bioengineering (K.L., B.M.E.), Henry Samueli School of Engineering and Applied Science; and UCLA Neuro-Oncology Program (T.F.C., A.L., P.L.N., B.M.E.), University of California, Los Angeles, Los Angeles, California; Departments of Radiological Sciences (K.L., W.B.P., B.M.E.), Biomedical Physics (B.M.E.), Neurology (A.L., P.L.N.), and Neurosurgery (L.M.L.), David Geffen School of Medicine, University of California, Los Angeles, Los Angeles, California; and Department of Diagnostic Imaging (J.L.B.), Rhode Island Hospital and Alpert Medical School of Brown University, Providence, Rhode Island.

This work was supported by the American Cancer Society Research Scholar Grant RSG-15-003-01-CCE (B.M.E.); National Institutes of Health/National Cancer Institute R21CA167354 (B.M.E.); UCLA Jonsson Comprehensive Cancer Center Seed Grant (B.M.E.); National Brain Tumor Society Research Grant (B.M.E.); Siemens Healthcare Research Grant (B.M.E.); Art of the Brain (T.F.C.); Ziering Family Foundation in memory of Sigi Ziering (T.F.C.); Singleton Family Foundation (T.F.C.); Clarence Klein Fund for Neuro-Oncology (T.F.C.); National Institutes of Health National Institute of General Medical Sciences training grant, GM08042 (K.L.); and the University of California, Los Angeles Medical Scientist Training Program (K.L.). distinguishing recurrent tumor from pseudoprogression, ${ }^{6,7}$ differentiating tumor regression from pseudoresponse, ${ }^{8}$ and assessing overall treatment response. ${ }^{9,10}$ Relative CBV is typically calculated by integrating the dynamic first-pass change in the transverse relaxation rate $\left(\Delta R_{2}{ }^{*}\right)$ resulting from bolus injection of a gadolinium-based contrast agent, which transiently causes a dose-dependent change in the magnetic susceptibility of blood. ${ }^{11}$ This technique mimics the classic indicator-dilution theory, ${ }^{12}$ which assumes intravascular compartmentalization of injected contrast agent "tracer." However, common gadolinium-based contrast agents extravasate in lesions with blood-brain barrier disruption, ${ }^{13}$ including malignant gliomas. The exchange of con-

\footnotetext{
Please address correspondence to Benjamin M. Ellingson, PhD, UCLA Brain Tumor Imaging Laboratory, Departments of Radiological Sciences and Psychiatry, David Geffen School of Medicine, University of California, Los Angeles, 924 Westwood Blvd, Suite 615, Los Angeles, CA 90024; e-mail: bellingson@mednet.ucla.edu; @ben_ellingson

- Indicates open access to non-subscribers at www.ajnr.org

http://dx.doi.org/10.3174/ajnr.A4759
} 
trast agent between the intravascular and the extravascular extracellular space, which is the objective measurement in dynamic contrast-enhanced (DCE) MR imaging, ${ }^{14-16}$ contaminates the desired DSC MR imaging signal, depending on pulse sequence parameters and underlying tumor biology. ${ }^{17}$

A popular model-based DSC MR imaging leakage-correction method proposed by Weisskoff and Boxerman ${ }^{2,18,19}$ linearly fits measured $\Delta R_{2}{ }^{*}(t)$ to 2 constant functions derived from the average relaxation rate in nonenhancing tissue, one of which is permeability-weighted. Deviation from the reference function is used to derive corrected rCBV for each voxel. A limiting assumption of this approach is that contrast agent reflux from the interstitial space back to blood plasma is negligible within the time frame of DSC MR imaging signal acquisition ( $\sim 2$ minutes). However, standard models quantifying contrast agent exchange between blood plasma and the interstitium (ie, DCE MR imaging ${ }^{14}$ ) use 2-compartment pharmacokinetics to account for bidirectional transport of contrast agent. We hypothesized that incorporating bidirectional contrast agent transport into the original DSC MR imaging signal model improves rCBV estimates in brain tumors. To test this hypothesis, we compared model-based DSC MR imaging leakage-correction methods with and without consideration of bidirectional transport by using simulations and clinical application to high-grade gliomas.

\section{MATERIALS AND METHODS \\ Patients}

We studied 24 sequential patients with histologically proved glioblastoma multiforme (GBM) treated with maximal surgical resection followed by radiation therapy and concurrent temozolomide and both DSC MR imaging and DCE MR imaging performed at initial tumor progression. Of these, 2 patients illustrated no bolus of contrast during the DSC acquisition and 1 DSC dataset was corrupted by significant motion. Thus, 21 patients ( 15 men; mean age, 54 years; range, 30-73 years) were included in the final cohort. Progression was defined prospectively by the treating neuro-oncologists if subsequent scans showed $>2$ sequential months of increasing contrast enhancement and worsening mass effect or evidence of neurologic decline. Specifically, progression was defined as $\geq 25 \%$ increase in the sum of enhancing lesion volumes, new enhancing lesions of $>1 \mathrm{~cm}$ in maximum dimension, an unequivocal qualitative increase in nonenhancing tumor, or an unequivocal new area of non-contrast-enhancing tumor. Additionally, progression must have occurred $>3$ months following completion of radiation therapy. All participants gave informed written consent to have both DSC MR imaging and DCE MR imaging data collected. All procedures complied with the principles of the Declaration of Helsinki and were approved by the institutional review board at University of California, Los Angeles.

\section{DSC MR Imaging and DCE MR Imaging}

We retrospectively reviewed DSC MR imaging and DCE MR imaging scans (3T, Magnetom Trio or Magnetom Skyra; Siemens, Erlangen, Germany), acquired in the same scan session in all 21 patients. T1 maps were generated from 5 precontrast T1-weighted images (flip angles $=5^{\circ}, 10^{\circ}, 15^{\circ}, 20^{\circ}, 30^{\circ}$ ) before DCE MR imaging (3D spoiled gradient-echo sequence, 16 sections, 130 time points, 5 -second time resolution, TE/TR $=1.87 / 5 \mathrm{~ms}, 25^{\circ}$ flip angle, 3-mm section thickness, $256 \times 192$ matrix, 24-cm FOV). The DCE MR imaging was acquired for $\sim 10$ minutes, which was the waiting time between preload and DSC contrast injections for this study. Contrast agent bolus $(0.1 \mathrm{mmol} / \mathrm{kg}$ ) (gadopentetate dimeglumine, Magnevist; Bayer HealthCare Pharmaceuticals, Wayne, New Jersey) was injected after 10-13 baseline images, serving as a preload ${ }^{13}$ for DSC MR imaging (gradient-echo EPI, $\mathrm{TE} / \mathrm{TR}=32 / 1840 \mathrm{~ms}, 35^{\circ}$ flip angle, 120 time points, bolus injection after $20-25$ baseline images, 9-20 sections, 5-mm section thickness, $128 \times 128$ matrix size, $24-\mathrm{cm}$ FOV). The same amount of contrast agent was used for the DSC MR imaging studies. Conventional postcontrast T1-weighted imaging was subsequently performed. Patients were excluded if DCE MR imaging or DSC MR imaging was corrupted by motion or technical error.

\section{Image Registration and ROI Selection}

All conventional and DCE MR images for each subject were registered to baseline DSC MR images by using a 12-df affine transformation with a mutual information cost function (FSL; http:// www.fmrib.ox.ac.uk/fsl). If required, manual alignment was subsequently performed (tkregister2, Freesurfer; http://surfer. nmr.mgh.harvard.edu/). Contrast-enhancing tumor ROIs were defined in 3D by using custom scripts (Analysis of Functional Neuro Images [AFNI]; http://afni.nimh.nih.gov/afni), excluding hemorrhage, large vessels, and central necrosis, followed by manual editing to exclude nonlesion voxels. ${ }^{20}$ Tumor sizes ranged from 2.8 to $106.6 \mathrm{~mL}$, with an average enhancing volume of $40.1 \pm 28.4 \mathrm{~mL}$. Spheric ROIs of $1.6 \mathrm{~mL}$ were also selected in normal-appearing, contralateral white matter for $\mathrm{rCBV}$ normalization.

\section{Computation of DSC MR Imaging rCBV}

All simulations and calculations were performed in Matlab (MathWorks, Natick, Massachusetts) by using custom scripts. Uncorrected rCBV was calculated from trapezoidal integration of the original DSC MR imaging relaxation rate-time curve, $\Delta \hat{R}_{2}^{\star}(t)$. The whole-brain average relaxation rate for nonenhancing voxels (Equations 3 and 4, all equations are in the Appendix) was used for both the original Boxerman-Weisskoff model ${ }^{19}$ (BW model) and the new bidirectional exchange model (bidir model). Linear least-squares optimization was used to determine the free parameters for both the bidir-model (via Equation 7) and the BW model (Equation 5, with $\mathrm{k}_{\mathrm{ep}}=0$ ) algorithms, and the corrected rCBV was computed from Equation 8 . The average run-time per patient in Matlab was $19.5 \pm 6.7$ seconds for the bidir model and $18.3 \pm$ 6.2 seconds for the BW model (3.2-GHz Intel Core i5, $32 \mathrm{~GB}$ RAM). Tumor rCBV for each method was subsequently normalized to median $\mathrm{rCBV}$ within the normal-appearing white matter ROI.

\section{Simulation of DSC MR Imaging rCBV}

The whole-brain average relaxation rate, $\Delta \bar{R}_{2}^{\star}(t)$, was chosen from a sample patient and corresponds to the curve with $\mathrm{K}_{1}=1, \mathrm{~K}_{2}=$ 0 , and $\mathrm{k}_{\mathrm{ep}}=0 . \mathrm{K}_{2}=0.05$ (adding T1-dominant leakage) with $\mathrm{k}_{\mathrm{ep}}=0$ was set to simulate the BW model. A nonzero $\mathrm{k}_{\mathrm{ep}}(0.002$ or $0.005)$ was used to simulate the bidir model of $\Delta \hat{R}_{2}^{\star}(t)$. For $\mathrm{k}_{\mathrm{ep}}=$ 0.1 , the simulation is reflective of the correction of relaxation rate curves at "arterylike" voxels.

AJNR Am J Neuroradiol 37:1440-46 Aug 2016 www.ajnr.org 1441 
Total Leakage-Contaminated Relaxation Rate

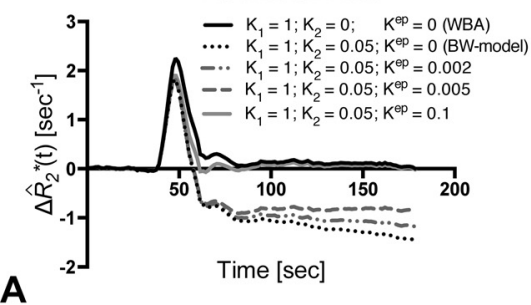

Sample Model Fits to Contaminated Relaxation Rate Curve

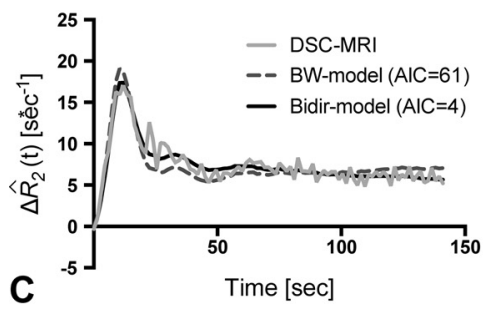

Interstitial Leakage

Relaxation Rate

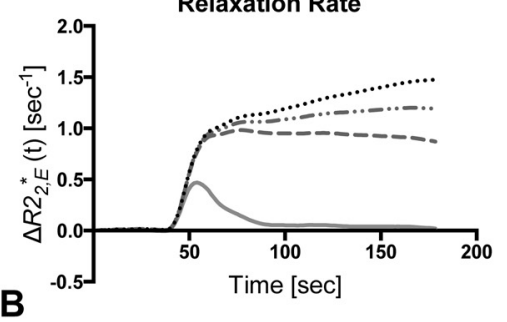

Comparison Between DCE-MRI and Interstitial Leakage Relaxation Rate Curves

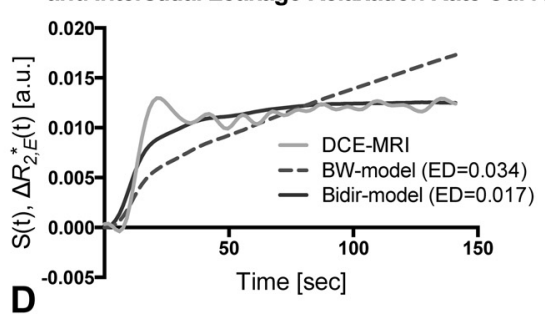

FIG 1. Sample simulated model results for all patients with GBM. A, Total leakage-contaminated relaxation rate and the component from interstitial leakage $(B)$ for T1-dominant leakage-associated relaxation enhancement. Whole-brain average relaxation rate (WBA) is simulated with $\mathrm{K}_{2}=$ 0 and $\mathrm{k}_{\mathrm{ep}}=0 . \mathrm{K}_{\mathrm{e}}=0$ with nonzero $\mathrm{K}_{2}$ simulates the $\mathrm{BW}$ model. Inclusion of a washout term (nonzero $\mathrm{k}_{\mathrm{ep}}$ ) in the bidir model yields less rise in $\Delta R_{2, \mathrm{E}}^{*}(t)$ and closer approximation of the tail of $\Delta \hat{R}_{2}^{\star}(t)$ to WBA. $C$, The bidir model fit to the sample leakage-contaminated relaxation rate curve has substantially improved AIC compared with the BW model for T2*-dominant leakage-associated relaxation enhancement in a patient with GBM. D, The standardized interstitial leakage relaxation rate from the bidir model better tracks standardized DCE MR imaging signal than the BW model for the tumor voxel used in $C$, with substantially improved Euclidean distance.

\section{Goodness of Fit Analysis}

For each enhancing tumor voxel for all patients, we computed the Akaike Information Criterion (AIC) between the leakage-contaminated relaxation rate $\Delta \hat{R}_{2}^{*}(t)$ (Equation 1) and its model fit (Equation 5) for the BW model and bidir-model:

$$
A I C=n \times \ln (R S S / n)+2(p+1),
$$

where $n$ is the number of fitted time points (injection to the end of the DSC MR imaging acquisition), RSS is the sum of the squared residuals, and $p$ is the number of free parameters ( 2 for the BW model, 3 for the bidir-model). ${ }^{21}$ Differences in the BW model and bidir model AIC were calculated for all voxels with $\mathrm{k}_{\mathrm{ep}}>0$.

We also computed the Euclidean distance (square root of the sum of the squared differences) between the interstitial leakage relaxation rate curves, $\Delta R_{2, \mathrm{E}}^{*}(t)$, generated by the BW model and bidir model corrections and the DCE MR imaging signal, in which the DCE MR imaging signal was upsampled from a 5-second resolution to a 1.8 -second resolution to match that of the DSC MR imaging data via linear interpolation by using the Matlab function "resample." Because interstitial leakage relaxation rate curves and DCE MR imaging signals have units of $1 / \mathrm{s}$ and $\mathrm{mM}$, respectively, both were standardized to an area under the curve equal to unity and were vectorized for computation of the Euclidean distance. Higher AIC and Euclidean distance imply worse fits. Two-sample $t$ tests were used to compare whether the AIC and Euclidean distance measurements were significantly different between the 2 leakage-correction methods.

\section{Postprocessing of DCE MR Imaging}

DCE MR imaging biomarkers, $\mathrm{k}_{\mathrm{ep}}$ and contrast transfer coefficient ( $K^{\text {trans)}}$, were derived via a fit to the model of Tofts and Kermode. ${ }^{14}$ As described, the temporal resolution of the DCE MR imaging data was upsampled to match the DSC MR imaging data. For the DCE MR imaging analysis, the "whole-brain average" served as the arterial input function for the DCE model fit. This was done to mirror the DSC bidir model analysis, in which the "whole-brain average" effectively serves as the arterial input function. Voxels with highly fluctuating time courses in either the DSC or DCE images were eliminated from the analysis.

\section{Correlation between DSC- and DCE-Derived Imaging Biomarkers}

DSC MR imaging biomarkers, $\mathrm{k}_{\mathrm{ep}}$ and rCBV, were derived as described in the Appendix. Voxelwise Pearson correlation coefficients between the DSC- and DCEderived parameters were performed in Matlab within contrast-enhancing tumor only, for each patient independently. In this study, we report means and SDs of the correlation coefficients from all 21 patients.

\section{RESULTS}

\section{Simulation of the Bidir Model}

Figure 1 compares the simulated total leakage contaminated relaxation rate, $\Delta \hat{R}_{2}^{*}(t)$, (Fig $\left.1 A\right)$ and the component from interstitial leakage, $\Delta R_{2, \mathrm{E}}^{*}(t)$, (Fig $\left.1 B\right)$ for various conditions according to the Tofts and Kermode model, ${ }^{14}$ assuming T1-dominant leakage-associated relaxation enhancement. For the BW model, $\Delta R_{2, \mathrm{E}}^{*}(t)$ rises with time in the absence of washout. For nonzero $\mathrm{k}_{\mathrm{ep}}$, there is less rise in $\Delta R_{2, \mathrm{E}}^{*}(t)$ and closer approximation of the tail of $\Delta \hat{R}_{2}^{*}$ $(t)$ to $\Delta \bar{R}_{2}^{*}(t)$, reflecting tumors with different contrast agent pharmacokinetics. For $\mathrm{k}_{\mathrm{ep}}=0.1$, the tail of $\Delta R_{2, \mathrm{E}}^{*}(t)$ approaches zero, but because the first-pass of $\Delta \hat{R}_{2}^{\star}(t)$ differs from that of $\Delta \bar{R}_{2}^{\star}(t)$, correction of relaxation rate curves at "arterylike" voxels by using $\mathrm{K}_{1}$ and $\mathrm{K}_{2}$ is still required to achieve accurate $\mathrm{rCBV}$ estimates.

Figure $1 C$ plots sample $\Delta \hat{R}_{2}^{\star}(t)$, with $\mathrm{T} 2{ }^{\star}$-dominant leakageassociated relaxation enhancement for a representative patient, with superimposed BW model and bidir model fit relaxation rate curves. In this example, the BW model overestimates the first-pass curve, underestimates the second and third passes, and overestimates the tail. The bidir model better approximates $\Delta \hat{R}_{2}^{\star}(t)$ over all time points, visually, and has substantially improved the AIC, quantitating an improved fit to the total leakage-contaminated relaxation rate curve.

Figure $1 D$ plots standardized DCE MR imaging signal for the tumor voxel used in Fig $1 C$, with superimposed standardized interstitial leakage relaxation rate curves, $\Delta R_{2, \mathrm{E}}^{*}(t)$, from the BW model and bidir model. The standardized interstitial leakage re- 
laxation rate continually rises with time for the BW model, whereas it better tracks standardized DCE MR imaging for the bidir model, with a substantially improved Euclidean distance.

\section{Goodness of Fit Analysis}

Figure 2 plots the percentage of voxels in which the bidir model outperformed the BW model for AIC and Euclidean distance metrics in whole brain and tumor for the 21 patients with GBM. The bidir model had better AIC performance than the BW model in $>50 \%$ of whole-brain (mean, $71 \% \pm 6 \%, P<.0001$ ) and tumor (mean, $77 \% \pm 9 \%, P<.0001$ ) voxels in all patients, and better Euclidean distance performance in $>50 \%$ of whole-brain voxels (mean, $80 \% \pm 9 \%, P<.0001$ ) for all patients and in tumor voxels (mean, $62 \% \pm 17 \%, P=.0041$ ) for 17 of the 21 patients. All were statistically significant for a 1 -sample $t$ test with null hypothesis of $50 \%$.

\section{Correlation between DSC- and DCE-Derived Imaging Biomarkers}

We then performed a voxelwise correlation between the DSCderived imaging biomarkers from the bidirectional leakage-correction algorithm ( $\mathrm{k}_{\mathrm{ep}}$ and $\left.\mathrm{rCBV}\right)$ with the DCE-derived imaging

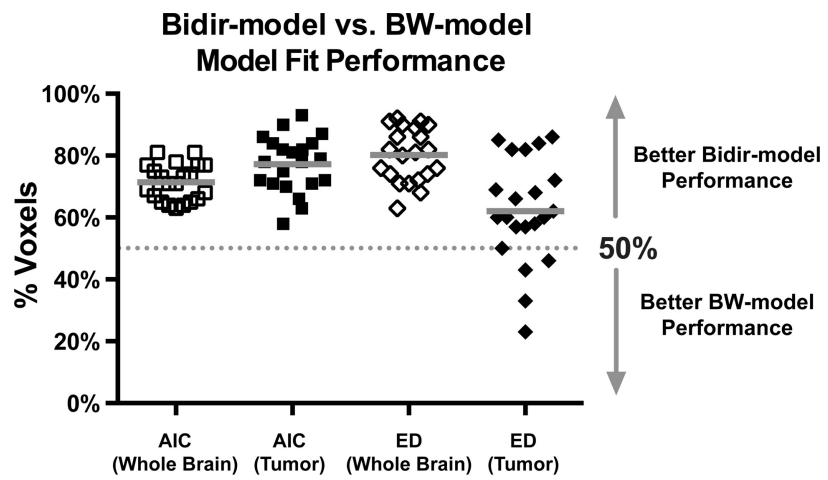

FIG 2. Percentage of voxels (with mean and SD) in which the bidir model outperformed the BW model on Akaike Information Criterion and Euclidean distance (ED) metrics within whole brain and tumor for all 21 patients with GBM. The gray line represents the group mean percentage of voxels. biomarkers $\left(\mathrm{k}_{\mathrm{ep}}\right.$ and $\left.K^{\mathrm{trans}}\right)$. The Pearson correlation coefficient between the $2 \mathrm{k}_{\mathrm{ep}}$ measurements was $0.74 \pm 0.13$ across the 21 patients, with a weak correlation between the Pearson correlation coefficient and tumor size $(r=0.11)$. Figure 3 demonstrates an example of the correlation between DSC- and DCE-derived $\mathrm{k}_{\mathrm{ep}}$. A correlation test was performed between the bidirectional modelderived $\mathrm{rCBV}$ and DCE-derived $K^{\text {trans }}$, with a moderate correlation of $0.49 \pm 0.22$. A moderate correlation was also found between $\mathrm{rCBV}$ and plasma volume fraction $(\mathrm{vp})$ at $0.54 \pm 0.12$. Finally, the correlation between the same rCBV and $\mathrm{k}_{\mathrm{ep}}$ was $r=$ $0.29 \pm 0.26$. The average $K^{\text {trans }}$ value was $0.0015 \pm 0.0018 \mathrm{sec}-$ onds $^{-1}\left(0.09 \pm 0.11\right.$ minutes $\left.^{-1}\right)$, DCE $_{\text {ep }}$ was $0.0050 \pm 0.0023$ seconds $^{-1}\left(0.30 \pm 0.14\right.$ minutes $\left.^{-1}\right)$, DSC $\mathrm{k}_{\mathrm{ep}}$ was $0.0057 \pm$ 0.0042 seconds $^{-1}\left(0.34 \pm 0.25\right.$ minutes $\left.^{-1}\right)$, vp was $0.01 \pm 0.01$, and $\mathrm{rCBV}$ was $1.98 \pm 1.24$.

\section{Difference in rCBV between the Bidir Model and BW Model}

Figure 4 compares rCBV maps processed without leakage correction and with the BW model or bidir model, in 2 different patients with GBM, one with T1-dominant leakage $\left(\mathrm{K}_{2}>0\right)$ on average in contrast-enhancing tumor voxels and the other with $\mathrm{T} 2{ }^{*}$-dominant leakage $\left(\mathrm{K}_{2}<0\right)$. For all patients, average uncorrected $\mathrm{rCBV}$ was $1.98 \pm 1.24$, the average BW model-corrected $\mathrm{rCBV}$ was $1.59 \pm 0.89$, and the average bidir model-corrected rCBV was $1.35 \pm 0.80$. The average difference between BW model-corrected and the bidir model-corrected $\mathrm{rCBV}$ was $16.6 \% \pm 14.0 \%$. A closer inspection of the $\mathrm{T} 2{ }^{\star}$-dominant-versus-T1-dominant voxels (as defined by a negative or positive $\mathrm{K}_{2}$, respectively) revealed that the difference between the 2 correction methods in $\mathrm{T} 2{ }^{\star}$-dominant voxels was $37.7 \% \pm 42.6 \%$, while the same metric for T1-dominant voxels was $5.8 \% \pm 3.4 \%$.

\section{DISCUSSION}

By incorporating the Tofts and Kermode model into the singleecho DSC MR imaging relaxation rate equation, we developed an improved postprocessing leakage-correction method accounting for bidirectional contrast agent transport between the intravascular and interstitial spaces that commonly occurs in angiogenic

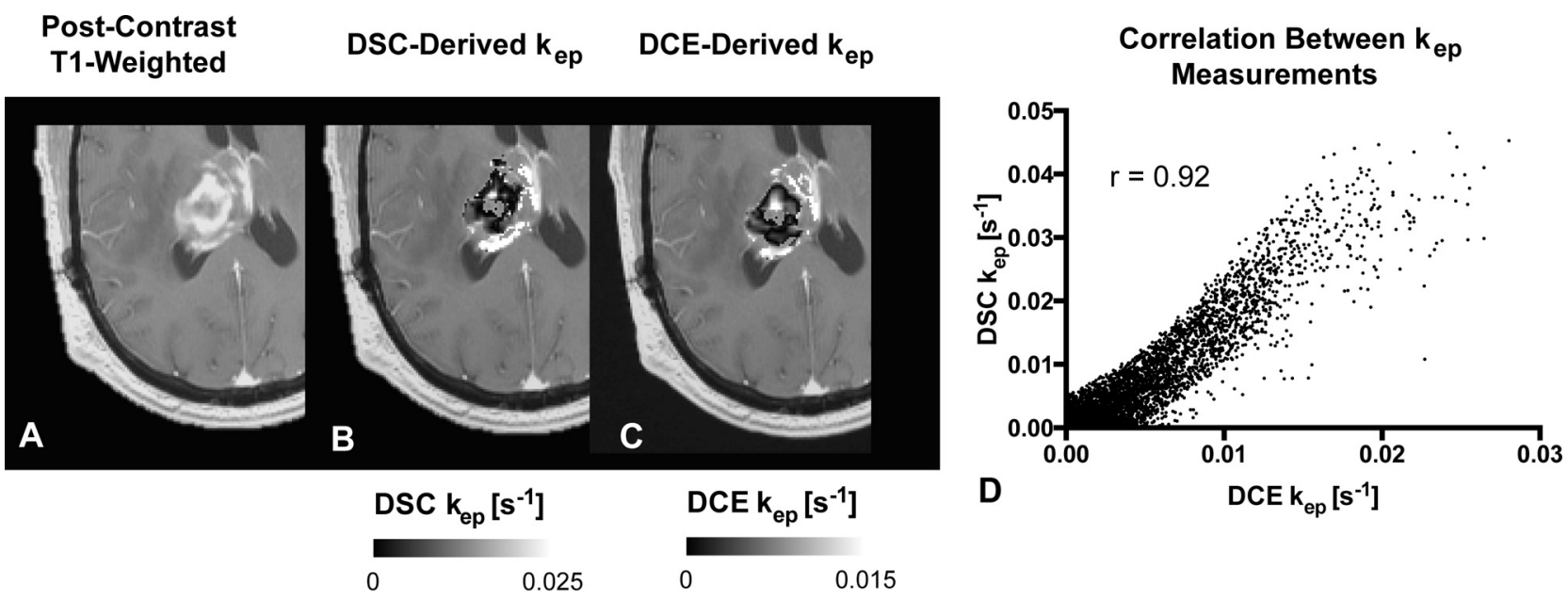

FIG 3. Comparison between DSC- and DCE-derived $\mathrm{k}_{\mathrm{ep}}$ measurements within tumor. $A$, Example of anatomic MR imaging of a patient with recurrent glioblastoma. $B$, DSC-derived $\mathrm{k}_{\mathrm{ep}}$ measurements within the tumor. C, Corresponding DCE-derived $\mathrm{k}_{\mathrm{ep}}$ measurements. D, Scatterplot between $B$ and $C$ demonstrates high correlation $(r=0.92)$ for this tumor. Note that areas of low $k_{e p}$ are similar in both DSC- and DCE-derived maps. 


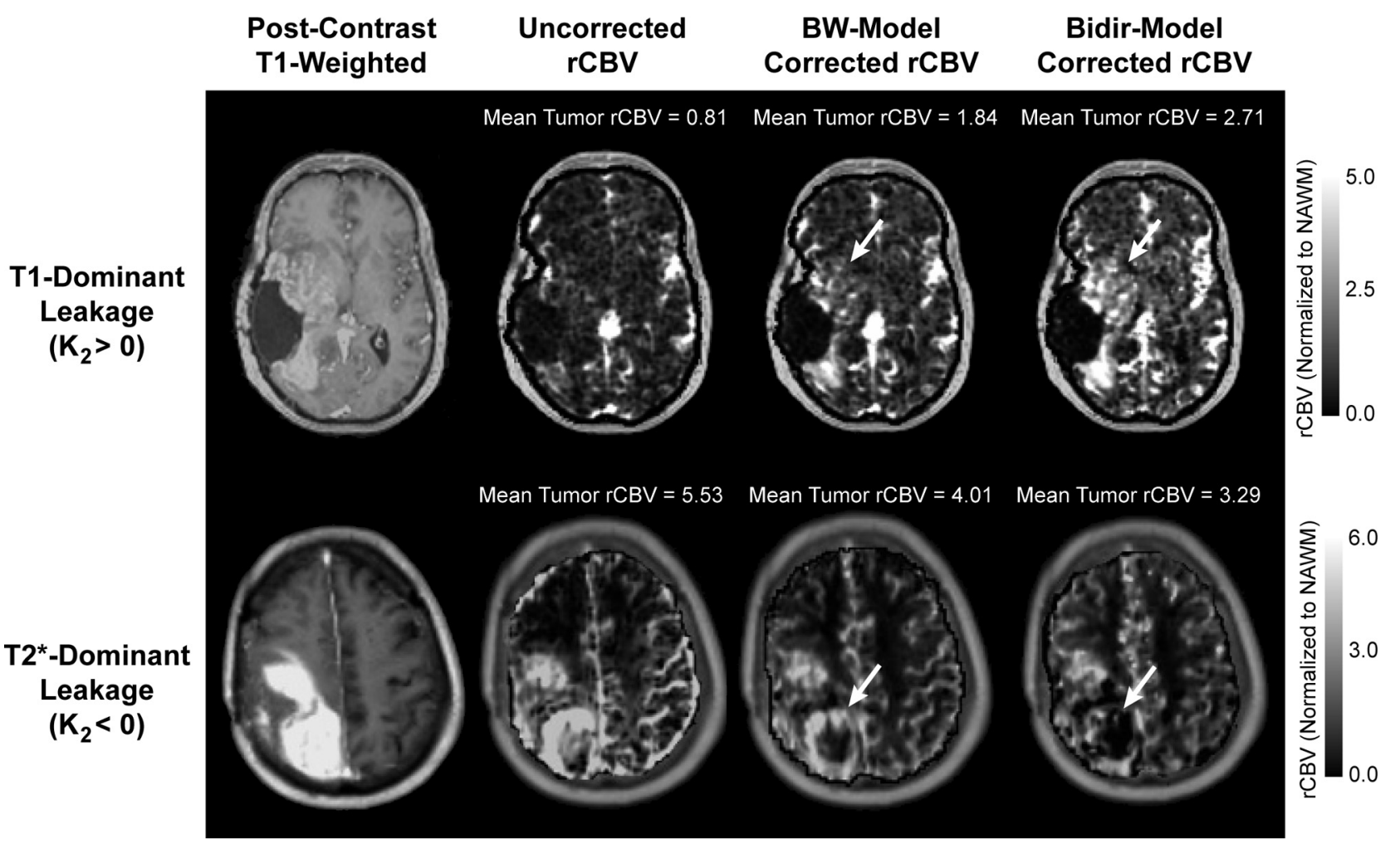

FIG 4. Comparison of uncorrected, BW model-corrected, and bidir model-corrected rCBV in a GBM with T1-dominant leakage on average in contrast-enhancing voxels (first row) and a GBM with T2*-dominant leakage (second row). For T1-dominant leakage, mean tumor rCBV is underestimated by using the BW model compared with the bidir model, with the converse true for T2*-dominant leakage. Arrows depict regions of the tumor with large changes in estimated rCBV between leakage-correction models.

high-grade gliomas. Our results demonstrate the importance of considering the interstitial washout term, even when modeling the relaxation rate changes during short image acquisitions. For instance, in the simulation, we observed differences between the bidir model and the BW model fits to relaxation rate data in highgrade gliomas in the first-pass curve (as early as 10-20 seconds after injection). Furthermore, inclusion of a washout term in the bidir model alleviates the error in relaxation rate estimates for arteries and normal brain introduced by conventional models constrained to increasing contrast agent concentration with time in all tissues.

Our results suggest that the conventional BW model undercorrects rCBV, with insufficiently increased and decreased rCBV compared with uncorrected $\mathrm{rCBV}$ in $\mathrm{T} 1$-dominant and $\mathrm{T} 2^{\star}$ dominant leakage scenarios, respectively. Furthermore, because the low flip angle DSC MR imaging protocol was largely $\mathrm{T}^{\star}{ }^{*}$ dominant and the largest discrepancies between the bidir model and BW model estimates of rCBV existed for $\mathrm{T} 2^{*}$ dominant voxels, our results suggest that the bidir model may be particularly advantageous over the BW model for correcting the residual T2* effects frequently encountered in dual-echo gradient-echo acquisitions. This algorithm can be performed without a substantial increase in postprocessing computation time over the unidirectional model; therefore, the bidirectional model can simply replace the previous model in routine clinical work and for evaluating tumor grade, distinguishing pseudoprogression from true progression, and evaluating treatment response.

Several postprocessing leakage-correction techniques have previously been proposed. ${ }^{22,23}$ The method by BoxermanWeisskoff, ${ }^{2,18,19}$ which linearly fits measured $\Delta \hat{R}_{2}^{\star}(t)$ to 2 constant functions derived from the average relaxation rate in nonenhancing tissue, can be applied quickly to conventional single-echo (spin-echo or gradient-echo) acquisitions and contrast agent injection schemes. Improved correlation of $\mathrm{rCBV}$ with glioma grade compared with uncorrected $\mathrm{rCBV}^{19}$ provides anecdotal evidence of the benefit of the BW model, which has also been shown to improve correlation of gadolinium-based rCBV measures over those obtained by using the intravascular magnetic iron oxide nanoparticles agent as a criterion standard. ${ }^{24}$

Bjornerud et $\mathrm{al}^{25}$ proposed a method that reduces the sensitivity of rCBV correction to mean transit time that could be combined with the bidir model scheme. Most interesting, Schmiedeskamp et $\mathrm{al}^{23}$ used a multiecho, gradient-echo, spin-echo acquisition scheme to correct for $\mathrm{T} 1$ and $\mathrm{T} 2{ }^{*}$ leakage by using a backflow term; however, results were highly dependent on literature values for $r_{2, \mathrm{E}}^{*}$ and $r_{2, \mathrm{P}}^{*}$ the $\mathrm{T} 2^{\star}$ relaxation effects of gadolinium in the extravascular space and plasma, respectively, which can vary quite substantially depending on the literature source. Additionally, Quarles et $\mathrm{al}^{17}$ suggested that these values could vary from tumor to tumor, depending on physiologic factors such as interstitial, vascular, and cell volume fractions and vessel and cell size. An advantage of the bidir model correction method is the lack of assumptions for $r_{2, \mathrm{E}}^{*}$ and $r_{2, \mathrm{P}}^{*}$. All of these leakage-correction algorithms aim to isolate the relaxation rate due to the residual intravascular contrast agent by eliminating the $\mathrm{T} 1$ - and $\mathrm{T} 2{ }^{\star}$-related contributions to the relaxation rate from the extravasated contrast agent. They do not "add 
back" $\mathrm{T} 2{ }^{*}$ relaxation that would have been realized had the extravasated contrast agent not left the plasma space, so "corrected rCBV" may still differ from that computed for a tumor with no vascular permeability, all other parameters (including true blood volume) being equal.

One potential limitation to this study is its retrospective design, which may have yielded a selection bias in the sample. Specifically, all patients were chosen because they failed standard therapy. Another potential limitation is the lack of correlation with a criterion standard, such as histology, or with CBV estimates by using intravascular agents such as iron oxide contrast agents. Moreover, AIC is a unitless quantity, which can compare relative goodness of fit between models but does not have a direct test to determine whether one model is significantly better than the other. Finally, the current study only included patients with glioblastoma; therefore, we were unable to recommend a threshold between low-grade and high-grade gliomas by using the new leakage-correction algorithm.

\section{CONCLUSIONS}

The bidir model more accurately corrects for the T1 or T2* enhancement arising from contrast agent extravasation due to blood-brain barrier disruption in high-grade gliomas by incorporating interstitial washout rates into the DSC MR imaging relaxation rate model. To this end, the bidir model may potentially improve patient diagnosis and evaluation of treatment response by more accurately estimating $\mathrm{rCBV}$ in DSC MR imaging.

\section{APPENDIX}

Following Equation A6 of Boxerman et al, ${ }^{19}$ the leakage-contaminated DSC MR imaging relaxation rate-time curve, $\Delta \hat{R}_{2}^{\star}(t)$, equals the intravascular contrast-driven transverse relaxation rate change, $\Delta R_{2}^{*}(t)$ plus $\Delta R_{2, \mathrm{E}}^{*}(t)$, a tissue-leakage term describing the simultaneous $\mathrm{T} 1$ and $\mathrm{T} 2^{\star}$ relaxation effects resulting from gadolinium extravasation:

1) $\Delta \hat{R}_{2}^{*}(t)=\Delta R_{2}^{*}(t)+\Delta R_{2, \mathrm{E}}^{*}(t)=\Delta R_{2}^{*}(t)$

$$
+\left[r_{2, \mathrm{E}}^{*}-\frac{T R}{T E} \times\left(\frac{E_{1}}{1-E_{1}}\right) \times r_{1}\right] C_{\mathrm{E}}(t),
$$

where $E_{1}=e^{-T R / T_{10}}, T_{10}$ is the precontrast tissue $T_{1}, r_{1}$ is the $T_{1}$ relaxivity of gadolinium, $C_{\mathrm{E}}(t)$ is the concentration of gadolinium in the extravascular extracellular space, and $r_{2, \mathrm{E}}^{*}$ represents the $\mathrm{T} 2{ }^{*}$ relaxation effects of gadolinium extravasation, as described by Quarles et $\mathrm{al}^{17}$ and Schmiedeskamp et al. ${ }^{23}$ From the original Tofts and Kermode model describing bidirectional contrast agent flux between the intravascular and extravascular compartments, ${ }^{14}$ we can estimate the concentration in the extravascular space as:

$$
C_{\mathrm{E}}(t)=k^{\text {trans }} \otimes\left[C_{\mathrm{p}}(t) \times e^{-k_{\mathrm{ep}} t}\right],
$$

where $k^{\text {trans }}$ and $k_{\text {ep }}$ are the transfer coefficients for intra- to extravascular and extra- to intravascular contrast flux, respectively, and $C_{\mathrm{p}}(t)$ is the plasma contrast concentration. $C_{\mathrm{p}}(t)$ and $\Delta R_{2}{ }^{*}(t)$ can be defined as scaled versions of the whole-brain average relaxation rate in nonenhancing voxels, $\Delta \bar{R}_{2}^{\star}(t)^{19}$ :

$$
C_{\mathrm{p}}(t)=k \times \Delta \bar{R}_{2}^{*}(t)
$$

$$
\Delta R_{2}^{\star}(t)=K_{1} \times \Delta \bar{R}_{2}^{\star}(t) .
$$

Combining Equations 1-4 yields the following:

$$
\Delta \hat{R}_{2}^{*}(t)=K_{1} \times \Delta \bar{R}_{2}^{*}(t)-K_{2} \int_{0}^{t} \Delta \hat{R}_{2}^{*}(\tau) \times e^{-k_{\mathrm{ep}}(t-\tau)} d \tau,
$$

where

6) $K_{2}=\left[r_{2, \mathrm{E}}^{*}-\frac{T R}{T E} \times\left(\frac{E_{1}}{1-E_{1}}\right) \times r_{1}\right] \times k^{\text {trans }} \times k$.

$K_{1}, K_{2}$, and $k_{\text {ep }}$ (units of second ${ }^{-1}$ ) are the free parameters of Equation 5. In general, $K_{1}$ depends on CBV, vessel size, and other physiologic factors, while $K_{2}$ is related to vascular permeability. Substituting $k_{\mathrm{ep}}=0$, which occurs with no backflow of extravasated contrast agent, yields the original Boxerman-Weisskoff leakage-correction algorithm, where $K_{1}$ and $K_{2}$ are solved by linear least-squares fit to $\Delta \hat{R}_{2}^{*}(t) .{ }^{19}$ For the bidir model correction method, a linear least-squares fit to $K_{1}, K_{2}$, and $k_{\text {ep }}$ can be used with the methodology of Murase, ${ }^{26}$ as described by the following equation:

$$
\text { 7) } \begin{aligned}
\Delta \hat{R}_{2}^{\star}(t)=\left(K_{2}+k_{\mathrm{ep}} \times K_{1}\right) \int_{0}^{t_{\mathrm{k}}} \Delta \bar{R}_{2}^{\star}(\tau) d \tau-k_{\mathrm{ep}} \\
\quad \times \int_{0}^{t_{\mathrm{k}}} \Delta \hat{R}_{2}^{\star}(\tau) d \tau+K_{1} \times \Delta \bar{R}_{2}^{\star}(t) .
\end{aligned}
$$

Integrating the corrected relaxation rate-time curve yields the following expression for leakage-corrected rCBV:

8) $r C B V_{\text {corr }}=r C B V+K_{2} \int_{0}^{\mathrm{T}} \int_{0}^{\mathrm{t}} \Delta \bar{R}_{2}^{\star}(\tau) \times e^{-k_{\mathrm{epp}}(t-\tau)} d \tau d t$.

Disclosures: Timothy F. Cloughesy—UNRELATED: Consultancy: F. Hoffmann-La Roche; Genentech; Celgene, Tocagen, AbbVie, Novocure, Stem Cycle, Novartis, Nektar, Newgen, Notable Labs, Upshire-Smith, VBL. Albert Lai-UNRELATED: Consultancy: Genentech, F. Hoffmann-La Roche, Novocure. Linda Liau—UNRELATED: Grants/Grants Pending: Northwest Biotherapeutics, ${ }^{*}$ Comments: research grant. Whitney B. Pope—UNRELATED: Consultancy: Guerbet. *Money paid to the institution.

\section{REFERENCES}

1. Cha S, Knopp EA, Johnson G, et al. Intracranial mass lesions: dynamic contrast-enhanced susceptibility-weighted echo-planar perfusion MR imaging. Radiology 2002;223:11-29 CrossRef Medline

2. Aronen HJ, Gazit IE, Louis DN, et al. Cerebral blood volume maps of gliomas: comparison with tumor grade and histologic findings. $R a$ diology 1994;191:41-51 CrossRef Medline

3. Law M, Yang S, Wang H, et al. Glioma grading: sensitivity, specificity, and predictive values of perfusion MR imaging and proton MR spectroscopic imaging compared with conventional MR imaging. AJNR Am J Neuroradiol 2003;24:1989-98 Medline

4. Law M, Oh S, Babb JS, et al. Low-grade gliomas: dynamic susceptibility-weighted contrast-enhanced perfusion MR imagingprediction of patient clinical response. Radiology 2006;238:658-67 CrossRef Medline

5. Danchaivijitr N, Waldman AD, Tozer DJ, et al. Low-grade gliomas: do changes in rCBV measurements at longitudinal perfusionweighted MR imaging predict malignant transformation? Radiology 2008;247:170-78 CrossRef Medline 
6. Boxerman JL, Ellingson BM, Jeyapalan S, et al. Longitudinal DSCMRI for distinguishing tumor recurrence from pseudoprogression in patients with a high-grade glioma. Am J Clin Oncol 2014 Nov 26. [Epub ahead of print] Medline

7. Gahramanov S, Varallyay C, Tyson RM, et al. Diagnosis of pseudoprogression using MRI perfusion in patients with glioblastoma multiforme may predict improved survival. CNS Oncol 2014;3:389400 CrossRef Medline

8. Schmainda KM, Zhang Z, Prah M, et al. Dynamic susceptibility contrast MRI measures of relative cerebral blood volume as a prognostic marker for overall survival in recurrent glioblastoma: results from the ACRIN 6677/RTOG 0625 multicenter trial. Neuro Oncol 2015;17:1148-56 CrossRef Medline

9. Leu K, Enzmann DR, Woodworth DC, et al. Hypervascular tumor volume estimated by comparison to a large-scale cerebral blood volume radiographic atlas predicts survival in recurrent glioblastoma treated with bevacizumab. Cancer Imaging 2014;14:31 CrossRef Medline

10. LaViolette PS, Cohen AD, Prah MA, et al. Vascular change measured with independent component analysis of dynamic susceptibility contrast MRI predicts bevacizumab response in high-grade glioma. Neuro Oncol 2013;15:442-50 CrossRef Medline

11. Villringer A, Rosen BR, Belliveau JW, et al. Dynamic imaging with lanthanide chelates in normal brain: contrast due to magnetic susceptibility effects. Magn Reson Med 1988;6:164-74 CrossRef Medline

12. Meier P, Zierler KL. On the theory of the indicator-dilution method for measurement of blood flow and volume. J Appl Physiol 1954;6: 731-44 Medline

13. Paulson ES, Schmainda KM. Comparison of dynamic susceptibilityweighted contrast-enhanced MR methods: recommendations for measuring relative cerebral blood volume in brain tumors. Radiology 2008;249:601-13 CrossRef Medline

14. Tofts PS, Kermode AG. Measurement of the blood-brain barrier permeability and leakage space using dynamic MR imaging, 1: fundamental concepts. Magn Reson Med 1991;17:357-67 CrossRef Medline

15. Roberts HC, Roberts TP, Brasch RC, et al. Quantitative measurement of microvascular permeability in human brain tumors achieved using dynamic contrast-enhanced MR imaging: correlation with histologic grade. AJNR Am J Neuroradiol 2000;21:891-99 Medline
16. Ludemann L, Wurm R, Zimmer C. Pharmacokinetic modeling of Gd-DTPA extravasation in brain tumors. Invest Radiol 2002;37: 562-70 Medline

17. Quarles CC, Gochberg DF, Gore JC, et al. A theoretical framework to model DSC-MRI data acquired in the presence of contrast agent extravasation. Phys Med Biol 2009;54:5749-66 Medline

18. Donahue KM, Krouwer HG, Rand SD, et al. Utility of simultaneously acquired gradient-echo and spin-echo cerebral blood volume and morphology maps in brain tumor patients. Magn Reson Med 2000;43:845-53 Medline

19. Boxerman JL, Schmainda KM, Weisskoff RM. Relative cerebral blood volume maps corrected for contrast agent extravasation significantly correlate with glioma tumor grade, whereas uncorrected maps do not. AJNR Am J Neuroradiol 2006;27:859-67 Medline

20. Ellingson BM, Cloughesy TF, Lai A, et al. Quantitative volumetric analysis of conventional MRI response in recurrent glioblastoma treated with bevacizumab. Neuro Oncol 2011;13:401-09 CrossRef Medline

21. Burnham KP, Anderson DR. Model Selection and Inference: A Practical Information-Theoretic Approach. New York: Springer-Verlag; 1998

22. Quarles CC, Ward BD, Schmainda KM. Improving the reliability of obtaining tumor hemodynamic parameters in the presence of contrast agent extravasation. Magn Reson Med 2005;53:1307-16 Medline

23. Schmiedeskamp H, Andre JB, Straka M, et al. Simultaneous perfusion and permeability measurements using combined spin- and gradient-echo MRI. J Cereb Blood Flow Metab 2013;33:732-43 CrossRef Medline

24. Boxerman JL, Prah DE, Paulson ES, et al. The role of preload and leakage correction in gadolinium-based cerebral blood volume estimation determined by comparison with MION as a criterion standard. AJNR Am J Neuroradiol 2012;33:1081-87 CrossRef Medline

25. Bjornerud A, Sorensen AG, Mouridsen K, et al. T1- and T2*-dominant extravasation correction in DSC-MRI, part I: theoretical considerations and implications for assessment of tumor hemodynamic properties. J Cereb Blood Flow Metab 2011;31:2041-53 CrossRef Medline

26. Murase K. Efficient method for calculating kinetic parameters using T-1-weighted dynamic contrast-enhanced magnetic resonance imaging. Magn Reson Med 2004;51:858-62 Medline 